\title{
Asociación de materia orgánica y minerales en suelos Amazónicos: Un estudio espectroscópico
}

\section{Association of organic and mineral matter in amazon soils: A spectroscopy study}

\author{
José L. Clabel H. ${ }^{1,}{ }^{\star}$, Gustavo Nicolodelli ${ }^{1}$, Neide A. Felicio Perruci ${ }^{2}$, Vinícius D. N. Bezzon², \\ Débora M. B. P. Milori ${ }^{1}$ \\ ${ }^{1}$ Embrapa Instrumentação, São Carlos, SP-Brasil. \\ ${ }^{2}$ Departamento de Físico-Química, Instituto de Química - Univ. Estadual Paulista, Araraquara, SP-Brasil. \\ *E-mail: jclabel@ifsc.usp.br
}

Recibido el 4 de diciembre del 2106; aceptado el 20 de diciembre del 2016

DOI: https://doi.org/10.33017/RevECIPeru2016.0017/

\section{Resumen}

La asociación entre materia orgánica y materia mineral, en los diferentes perfiles de suelo amazónico de la región São Gabriel da Cachoeira, fueron estudiados. Con este propósito se colectaron nueve muestras de hasta una profundidad de $+290 \mathrm{~cm}$. El suelo entero fue estudiado por análisis elemental, difracción de rayos X, microscopia electrónica de barredura, y espectroscopia FTIR. De acuerdo con resultados obtenidos, el análisis elemental es un método satisfactorio para la cuantificación de Carbono, debido a su precisión y reproductibilidad. El análisis mineralógico del suelo entero mostro que el Cuarzo y Caolinita, es el principal constituyente para los horizontes estudiados de A1 para Bhs (profundidad de 0 a $214 \mathrm{~cm}$ ) y Tr para K2 (profundidad de $215 \mathrm{a}+290 \mathrm{~cm}$ ), respectivamente. Como consecuencia de los cambios estructurales, hay evidencias de mudanzas espectroscópicas que se reflejan en los grupos funcionales aromáticos y alifáticos.

Descriptores: Suelo amazónico, Espodosolo humilúvico, DRX

\begin{abstract}
The associations between organic and mineral matter, in the different Amazonian soils profile from the São Gabriel da Cachoeira region, were studied. We collected nine samples up to a depth of 4 meters. The whole soil was studied by elemental analysis, X-ray diffraction and FTIR spectroscopy. According to results, elemental analysis is a satisfactory method for the quantification of carbon, because of its precision and reproducibility. The mineralogical analysis of the whole soil showed that the Quartz and Kaolinite, is the main constituent for the studied horizons of A1 for Bhs (depth 0 to $214 \mathrm{~cm}$ ) and Tr for K2 (depth 215 to $290 \mathrm{~cm}$ ), respectively. As a consequence of the structural changes, there is evidence of spectroscopic changes that are reflected in the aromatic and aliphatic functional groups.
\end{abstract}

Keywords: Amazon soils, Spodosol humilúvic, DRX

\section{Introducción}

La región amazónica comprende una región extensa y heterogénea, en la cual factores climáticos, interacciones bióticas, formas de relevo 
y geomorfológico dan origen a una rica diversidad de tipos de suelo $[1,2]$. En suelos amazónicos los minerales y materia orgánica (MO) pueden formar asociaciones intrínsecas mediante diferentes interacciones [3,4]. Esta asociación de MO con la superficies de los minerales es principalmente investigado debido a la retención de $\mathrm{MO}$. Diferentes condiciones ambientales durante su formación y descomposición de la materia orgánica del suelo (MOS), tienen un gran impacto en la estructura del suelo (cantidad y composición) [5]. Es deseable, por tanto, distinguir los factores estructurales intrínsecos que gobiernan a tasa de descomposición de la MOS.

El Espodosol humiluvic son suelos minerales con alto grado de meteorización, predominantemente silicio o cuarzo en fracciones de arena o limo y con fertilidad química baja [6]. El tamaño de los constituyentes en Espodosol humiluvic son diferenciados de acuerdo al tamaño de partícula, esto es, tamaño de limo (>2 $\mu \mathrm{m}$ y $<63 \mu \mathrm{m})$ y tamaño de arcilla $(<2 \mu \mathrm{m})$. Cada tipo de agregado posee una formación propia que es reflejado en su tamaño, forma, composición y estabilidad. La morfología en los perfiles de Espodosol humiluvic presentan características diferenciadas de color, estructura, profundidad y espesura. En este sentido estas características controlaran la descomposición de la materia orgánica, además, afectara en el suelo la cantidad sino la calidad de la composición de la MOS $[1,6,7]$. La composición de MOS es definido en términos de grupos funcionales carboxílicos, hidroxilicos, alifáticos y aromáticos. Tales grupos son los responsables por las reacciones químicas y capacidad de absorber de materia orgánica de suelo. Silamikele [8], observa incremento de grupos funcionales aromáticos en suelos enteros con la profundidad.

En función a la asociación y características entre los perfiles de Espodosol humiluvic del suelo amazónico, este trabajo tiene como objetivo identificar y describir los minerales y orgánicos presentes envueltos en suelos amazónicos a partir de diferentes métodos de caracterización usando técnicas espectroscópicas, con la finalidad de estimar la asociación de la materia orgánica y mineral.

\section{Material y métodos}

\subsection{Identificación y preparación de las muestras}

El área de estudio se encuentra localizada en la propiedad del ministerio de aeronáutica en el estado de Amazonas, Brasil, a la margen izquierda del rio Negro a $26 \mathrm{Km}$ de São Gabriel da Cachoeira en las coordenadas geográficas $0^{\circ} 6$ '24,5" S latitud y $66^{\circ} 54^{\prime} 19,3$ " W longitud. Las muestras fueron obtenidas de diferentes perfiles de suelo.

El ambiente de la región es caracterizado por una temperatura media del mes $25^{\circ} \mathrm{C}$, con una precipitación anual media de $3000 \mathrm{~mm}$, siendo los meses de agosto a octubre y diciembre a mayo, con menor y mayor precipitación.[9] Las muestras fueron colectadas en una toposecuencia Espodosol en diferentes profundidades descritos en la Tabla 1. El proceso de las muestras colectadas fueron descritas en, El sitio de muestreo del suelo es clasificado como Espodosol Humiluvic, la cual es constituido por material mineral con presencia de horizonte espódico $B(B h$ y Bhs) [6]. El horizonte superior del perfil A (A1 y A2) son ricos en materia orgánica descompuesta 0 no. Abajo del horizonte A (horizonte orgánico), ocurre el horizonte $E$ (E1 y E2) es de carácter álbico gris y cuarzosos debido a la descomposición de la materia orgánica, lixiviación e migración del aluminio y fierro en presencia de materia orgánica para el horizonte sub superficial. Estas características indican que la podzolización en este perfil ocurre de forma intensa.

\subsection{Caracterización mineralógica y química}

En el laboratorio las muestras de suelo fueron cuarteadas, secas, molidas, colador (malla de/Sift Mesh 150-diametro de abertura $105 \mu \mathrm{m})$ y homogenizadas. Posteriormente, las muestras fueron sometidas a los análisis descritos a seguir.

El análisis químico para la determinación de la composición elemental (Carbono, Nitrógeno e Hidrogeno - $\mathrm{CHN}$ ) de las muestras fue realizado en tres replicas con una masa de $10 \mathrm{mg}$ de muestra, por combustión a $1000{ }^{\circ} \mathrm{C}$. Los análisis fueron realizados en el analizador elementar marca Perkin Elmer modelo 2400 e pesada directamente en capsulas de estaño, utilizando una microbalanza (Perkin Elmer AD-6 Auto Balance Controller).

La determinación de la composición mineral de las muestras de suelo entero fue realizado por un difractómetro de rayos X Rigaku (RINT2000), con radiación $\mathrm{Cu} \mathrm{K} \alpha$, fenda de divergencia de $14^{\circ}$, fenda de recepción de $0.30 \mathrm{~mm}$, monocromador curvo de grafite, porta muestra de aluminio con $20 \mathrm{~mm}$ de diámetro y paso de barredura de $0.02^{\circ}$. Las fases de los minerales fueron identificadas con 
el auxilio de Crystallographica Search-Match (CSM) Version 2.1. Los padrones de difracción fueron refinados usando el método de Rietveld por medio del programa de análisis estructural (TOPAS) [10].

Espectroscopia Infrarroja con Transformada de Fourier (FTIR) fue obtenida utilizado un espectrómetro VERTEX 70, operado en un intervalo de longitud de onda de 4000 a $400 \mathrm{~cm}^{-1} \mathrm{y}$ con una resolución de $1 \mathrm{~cm}^{-1}$, enlazado con un sistema computarizado, con el software OPUS 7.0. En torno de $1 \mathrm{mg}$ de muestra fue misturada con 90 mg de $\mathrm{KBr}$, finalmente prensadas en forma de pastilla, seguidamente almacenadas en desecador.

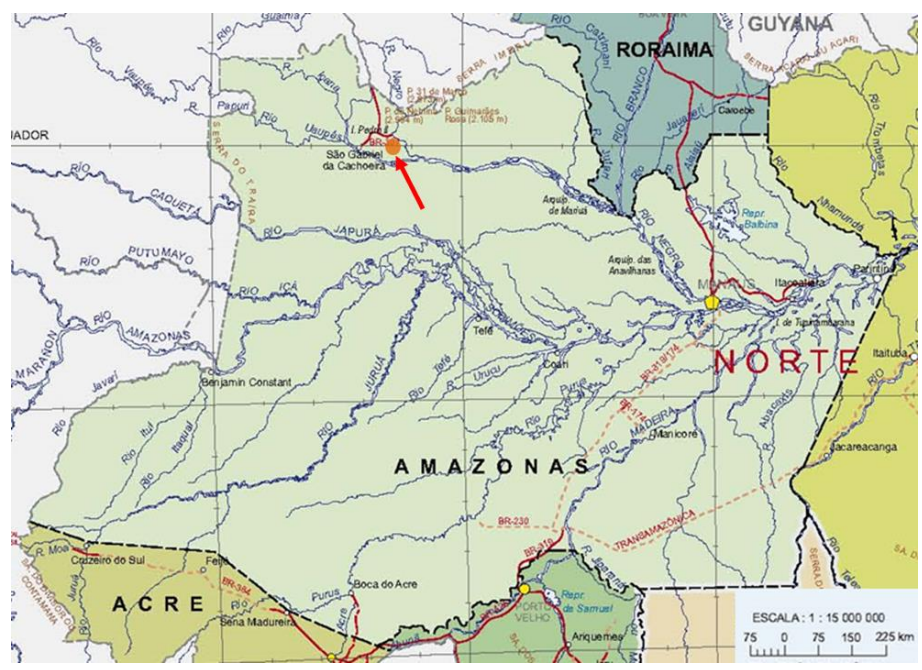

Figura 1: Localización del suelo en São Gabriel da Cachoeira, Amazonas-Brasil.

\section{Resultados y discusión}

El análisis elemental del suelo entero presento valores de acuerdo a la Tabla 1. Las informaciones de estos elementos son esenciales pero no conclusivas, particularmente, la razón/proporción de $\mathrm{C} / \mathrm{N}$ que es un indicador del grado de descomposición de la $\mathrm{MO}$, en la cual la competición entre los nutrientes es esencial para la actividad de microorganismos del suelo [11]. Stearman et. al. [12], indica que la relación $\mathrm{C} / \mathrm{N}$ es el grado de incorporación de $\mathrm{N}$ en la estrutuctura de la substancia húmica y el grado de humificación. En EH las medias de la composición de carbono en la profundidad de $0-5 \mathrm{~cm}$ fue de 7.9 $\mathrm{g} \mathrm{kg}^{-1}$ y decreciendo hasta $1.43 \mathrm{~g} \mathrm{~kg}^{-1}$ en la profundidad de $180-202 \mathrm{~cm}$. La tendencia de N, es similar al de C. Así mismo, la relación $\mathrm{C} / \mathrm{N}$ disminuye de 11 hasta 7 en la profundidad de 180$202 \mathrm{~cm}$. Un incremento ligero fue observada en el perfil del horizonte Bh y Bhs, donde la composición media de $C$ fue de 4.3 y 4.2 g $\mathrm{kg}^{-1}$, respectivamente. Los valores de la relación $\mathrm{C} / \mathrm{N}$ incrementan significativamente a partir de horizonte Bh. Por otro lado disminución de composición de $\mathrm{N}$ en el suelo refleja una mayor relación de $\mathrm{C} / \mathrm{N}$. Esta relación mayor puede llevar a una reducción en la mineralización y/o mudanza estructural [13], como será observado en los resultados obtenidos por DRX. Elevados valores de $\mathrm{H} \mathrm{y} \mathrm{H} / \mathrm{C}$ indican menor contenido de aros aromáticos en la estructura de suelo entero [14].

Tabla 1: Resultados del análisis elemental de las muestras de suelo entero.

\begin{tabular}{|c|c|c|c|c|c|c|c|}
\hline \multirow[t]{2}{*}{ Suelo } & \multirow[t]{2}{*}{ Hor. } & \multirow{2}{*}{$\begin{array}{l}\text { Profun. } \\
\text { (cm) }\end{array}$} & \multirow{2}{*}{$\begin{array}{c}\mathrm{pH} \\
(\mathrm{KCl})\end{array}$} & $\mathrm{C}$ & $\mathrm{H}$ & $\mathbf{N}$ & \multirow[t]{2}{*}{$\mathrm{C}: \mathrm{N}$} \\
\hline & & & & \multicolumn{3}{|c|}{$\left(\mathrm{g} \mathrm{kg}^{-1}\right)$} & \\
\hline \multirow{9}{*}{$\begin{array}{l}\text { 응 음 } \\
\text { 을 } \\
\text { 응 } \\
\text { 을 } \\
\text { 은 主 }\end{array}$} & A1 & $0-5$ & 5,8 & 7,90 & 1,00 & 0,76 & 11 \\
\hline & $\mathrm{A} 2$ & $5-30$ & 5,5 & 4,50 & 0,53 & 0,80 & 6 \\
\hline & E1 & $30-180$ & 5,8 & 1,43 & 0,90 & 0,56 & 3 \\
\hline & E2 & $180-202$ & 5,8 & 1,43 & 0,40 & 0,20 & 7 \\
\hline & $\mathrm{Bh}$ & $202-204$ & 5,2 & 4,30 & 0,90 & 0,33 & 13 \\
\hline & Bhs & $204-214$ & 5,5 & 4,20 & 1,23 & 0,30 & 14 \\
\hline & $\mathrm{Tr}$ & $214-245$ & 5,1 & 31,13 & 17 & 1,06 & 29 \\
\hline & K1 & $245-290$ & 5,4 & 8,26 & 14,96 & 0,56 & 15 \\
\hline & K2 & $290+$ & 5,4 & 3,23 & 13,70 & 0,43 & 8 \\
\hline
\end{tabular}

La difracción de rayos $X$ de suelo entero son mostrados en la Figura 2. Tres características importantes fueron determinadas a partir de los DRX: Primero, el refinamiento Rietveld de los horizontes de $A 1$ hasta $E 2$, indican que este solo entero $\mathrm{EH}$ exhibe una fase predominante de cuarzo $\left(\mathrm{SiO}_{2}\right)$, con estructura cristalina trigonal con espacio de grupo P 3221 de acuerdo al ICSD (Inorganic Crystal Structure Database) (ICSD N ${ }^{\circ}$ 16331). Segundo, el refinamiento Rietveld de los horizontes $\mathrm{Bh}$ y $\mathrm{Bhs}$ fue identificado la fase caolinita $\mathrm{Al}_{2}\left(\mathrm{Si}_{42} \mathrm{O}_{5}\right)(\mathrm{OH})_{4}$, anatase $\left(\mathrm{TiO}_{2}\right)$ y cuarzo $\left(\mathrm{SiO}_{2}\right)$ con estructura cristalina triclínica, tetragonal, trigonal y con espacio de grupo C1(ICSD $\mathrm{N}^{\circ}$ 80082), I 41/amdS (ICSD No 9852) y P 322 1 (ICSD $\left.N^{\circ} 16331\right)$, respectivamente. Tercero, para los horizontes $\mathrm{Tr}, \mathrm{K} 1$ y $\mathrm{K} 2$ el refinamiento estructural revelo dos tipos de fases caulinita-1A $\mathrm{Al}_{2}\left(\mathrm{Si}_{42} \mathrm{O}_{5}\right)(\mathrm{OH})_{4}$, y montmorillonite $\mathrm{Li}_{0.16}\left(\mathrm{Al}_{1.52} \mathrm{Fe}_{0.2}\right.$ $\left.\mathrm{Mg}_{0.28}\right)\left(\left(\mathrm{Si}_{7.8} \mathrm{Al}_{0.2}\right) \mathrm{O}_{18}(\mathrm{OH})_{2}\right)$ con estructura cristalina triclínica y monoclínica con espacio de grupo C1(ICSD No 87771) y C $12 / \mathrm{m}$, respectivamente. Las fases de los minerales están de acuerdo con los datos de la literatura. Estos resultados están de acuerdo a la estabilización de microagregados en el suelo y presentándose en las diferentes formas cristalinas.

El análisis cuantitativo en horizontes espódico Bh y Bhs fue observado el débil grado de 
desenvolvimiento de la estructura caolinita y anatase, siendo que el porcentaje de fase cuarzo $\left(\mathrm{SiO}_{2}-86.6 \%\right)$ es mayor que la fase de anatase $\left(\mathrm{TiO}_{2}-6.6 \%\right)$ y caolinita $\left(\mathrm{Al}_{2}\left(\mathrm{Si}_{42} \mathrm{O}_{5}\right)(\mathrm{OH})_{4}-6.8 \%\right)$, indicando que hay una amplia proporción de cuarzo en relación a caolinita e anatase. Margenot et. al. [15], afirma que en suelos espódico reconoce que presenta menor cantidad de mineral de caolinita y mayor concentración de grupos funcionales de hidroxilo en la superficie del mineral.



Figura 2: DRX representativos de las fracciones de suelo entero, presentando los siguientes minerales: cuarzo, caolinita, anatase, montmorillonite $y$ caolinita-1A.

Esta afirmación es coherente con los resultados en el presente estudio entre la estabilidad de los complejos órgano-minerales y la proporción de fase.
Estos resultados son importantes para entender la estabilidad de fase de los microagregados en suelo enteros de $E H$, la cual resulta de la interacción de la materia orgánica con los minerales presentes. Esta asociación entre materia orgánica y materia mineral ha contribuido a la retención de la materia orgánica, lo que representa un beneficio ambiental importante [1].

Como técnica complementaria al DRX, el FTIR nos permite analizar cualitativamente los grupos inorgánicos (hidroxilo, aromáticos, alifáticos etc). Los espectros de FTIR son mostrados en la Figura 3. Los horizontes superficiales y sub-superficiales mostrados A1 hasta E2 no muestran diferencia alguna en el espectro, pero, de Bh hasta K2 se observó cambios significativos entre los espectros de estos horizontes.
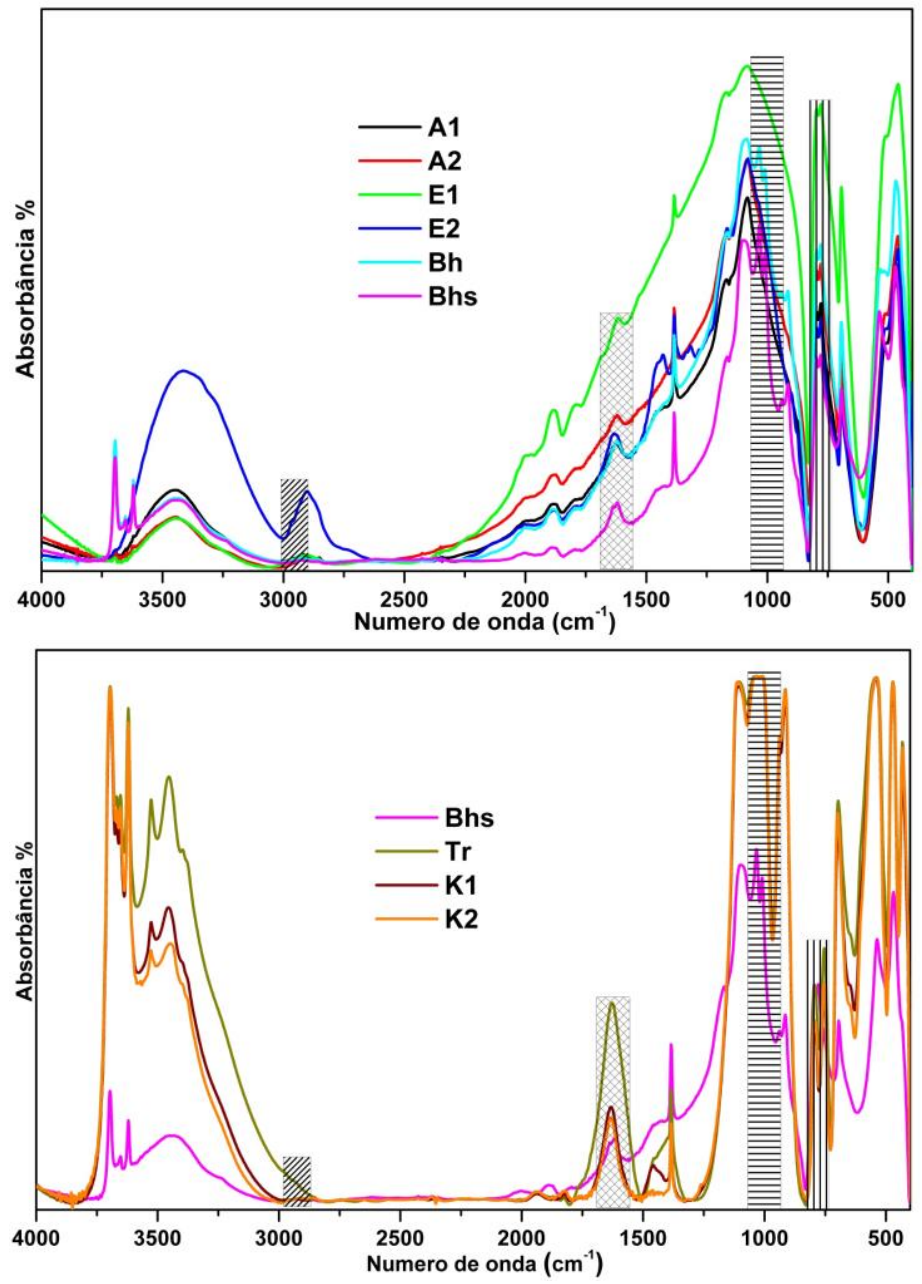

Figura 3: Espectro infrarrojo en suelos enteros de Espodosolo Humilúvico en diferentes profundidades.

En la banda de absorción de 3400 a $3380 \mathrm{~cm}^{-1}$, fueron observadas bandas de absorción alargadas desde A1 hasta E2 y menos alargadas Bh hasta 
K2. Esta región es característica de la absorción de vibración de estiramiento de grupos $\mathrm{OH}$ en puentes de hidrogeno. El tamaño de alargamiento de la absorción está relacionado a la con la fuerza de interacción de $\mathrm{H}$, es decir, cuanto mayor es la ligación de puentes de hidrogeno, mayor es el energía para estirar las ligaciones esto lleva a un alargamiento en la banda de absorción [16]. Sin embargo, Leonardo Barros Dobbss et. al. [17], asocia el alargamiento a las ligaciones de $\mathrm{H}$ con ácidos carboxílicos. Por otro lado, fue observada una absorción entre 3000 y $2800 \mathrm{~cm}^{-1}$ que fue atribuido a la presencia de grupos alifáticos $(\mathrm{C}-\mathrm{H})$. Así mismo, la absorción en $2914 \mathrm{~cm}^{-1}$, fue asociada a los grupos $\mathrm{CH}_{3}[18,19]$.

La absorción en 1720 y $1700 \mathrm{~cm}^{-1}$ fue asignado a grupos carbonilos y cetonas. La absorción entre 1725 y 1720 fue atribuido al estiramiento $\mathrm{C}=\mathrm{O}$ de grupos funcionales carboxílicos y cetonas [18,20]. En todos los horizontes fue observado una fuerte absorción en $1617 \mathrm{~cm}^{-1}$, excepto en los horizontes a partir de $\mathrm{Bh}$ a K2, donde la banda de absorción fue detectada en $1623 \mathrm{~cm}^{-1}$. Según Margenot et. al. [19], la banda de absorción $1610 \mathrm{~cm}^{-1}$ es atribuido a la vibración de $\mathrm{C}=\mathrm{C}$ aromáticos y entre 1645 y $1635 \mathrm{~cm}^{-1}$ la deformación axial de $\mathrm{C}=\mathrm{C}$ de los núcleos aromáticos.

Tales diferencias estructurales es debido a la interacción de la asociación entre la materia orgánica y la materia mineral del suelo entero lleva a diferencias en las propiedades físicas y químicas.

\section{Conclusiones}

La mineralogía de los suelos amazónicos presento tres características particulares: los horizontes A1 hasta $E 1$ se muestra homogénea como fue observado en los DRX, con predominio de cuarzo. En los horizontes Bh hasta Bhs son encontradas el cuarzo, anatase y caolinita. A partir del horizonte $\mathrm{Tr}$ hasta K2 presenta fase de montmorillonite y caolinita-1A. Estas composiciones mineralógicas reflejan las características de los suelos $E H$. Los espectros FTIR revelan que horizontes $A 1$ y $B h$ hasta K1 presenta mayor grado de descomposición de la materia orgánica.

\section{Agradecimientos}

Agradezco al equipo de apoyo de la EMBRAPA INSTRUMENTAÇÃO. Al Conselho Nacional de Desenvolvimento Científico e Tecnológico (CNPq) por el financiamiento de investigación con proceso CNPq 402624/2015-7. A la Fundação de Amparo a
Pesquisa do Estado de São Paulo (FAPESP) con proceso 2012/24349-0.

\section{Referencias}

[1] W.G. Sombroek, Soils of the Amazon region, Dordrecht, Boston. Lancaster., Netherlands, 1984.

[2] C.A. Quesada, J. Lloyd, L.O. Anderson, N.M. Fyllas, M. Schwarz, C.I. Czimczik, Soils of Amazonia with particular reference to the RAINFOR sites, (2011) 1415-1440.

[3] K.K. and G. Guggenberger, Mineral surfaces and soil organic matter, Eur. J. Soil Sci. 54 (2003) 219-236.

[4] G.O. South, W. Wagga, M.L. Campus, The effect of cation - anion interactions on soil $\mathrm{pH}$, (2015) 1054-1062.

[5] T. Lombricultores, N. Environment, O. De Rennes, S. Biologique, O. De Rennes, S. Biologique, A review of earthworm impact on soil function and, (2013) 161-182.

[6] EMBRAPA, Sistema Brasileiro de Classificação de Solos, Rio de Janeiro, 2013.

[7] N. Aran, Quantifying the effects of aggregation, particle size and components on the colour of Mediterranean soils, (2004) 551-565.

[8] I. Silamikela, Properties of Peat in Ombrotrophic Bigs Depending on the Humification Process, (2003).

[9] A.G. Silva, F. C. F.; Jesus, R. M.; Ribeiro, Vegetação, (1977) 297.

[10] Coelho A, Topas Academic., (2007).

[11] E. Luchese, E. B. ; Favero, L. O. B. ; Lenzi, Fundamentos da Química do Solo Teoria e Prática, $2^{\mathrm{a}}$ ed., Rio de Janeiro, 2002.

[12] and D.D.T. G. K. Stearman, R. J. Lewis, L. J. Tortorelli, Herbicide Reactivity of Soil Organic Matter Fractions in No-Tilled and Tilled Cotton, Soil Sci. Soc. Am. J. 1694 (1989) 1690-1694.

[13] M.W.I. Schmidt, C. Rumpel, I. Ko, Particle size fractionation of soil containing coal and combusted particles, (1999).

[14] E. Analysis, Organic Elemental Analysis of Soils - Understanding the Carbon-Nitrogen Ratio, (n.d.) 2-5.

[15] A. Margenot, IR Spectroscopy , Soil Analysis Applications, (2017).

[16] A.D.E. Eucalipto, C. Miranda, L.P. Canellas, M.T. Nascimento, Caracterizacão da matéria orgânica do solo em fragmentos de mata atlântica e em plantios abandonados de eucalipto, R. Bras. Ci. Solo. 31 (2007) 905- 
916.

[17] S.D.E.L. Brasileiros, L.B. Dobbss, V.M. Rumjaneck, M. Altoé, A. Carlos, X. Velloso, et al., Caracterizacao química e espectroscópica de ácidos húmicos e fúlvicos isolado da camada, (2009) 51-63.

[18] P. Larkin, Infrared and Raman Spectroscopy, Oxford, The Netherlands, 2011.

[19] S.J.P. A.J. Margenot, F.J Calderon, K.W.
Goyne, F.N.D. Mukome, Soil Analysis and Applications of IR Spectroscopy, Encycl. Spectrosc. Spectrom. (2016) 1-8.

[20] F.A.O. Ceretta, C.A.; Bayer, C.; Dick, D.P.; Martin-Neto, L. and Colnago, L.A. Métodos espectroscópicos. In: Santos, G.A.; Silva, L.P.; Canellas, L.P. and Camargo, Fundamentos da matéria orgânica do solo em ecossistemas tropicais e subtropicais., 2. ed., Metrópole, 2008. 